\title{
Efficacy of TachoSil, a Fibrin-Based Hemostat, for Anterior Lumbar Spine Surgery
}

\author{
Jo Watanabe, Seiji Ohtori, Sumihisa Orita, Kazuyo Yamauchi, Yawara Eguchi, Yasuchika Aoki, \\ Junichi Nakamura, Miyako Suzuki, Kazuhide Inage, Jun Sato, Yasuhiro Shiga, Koki Abe, \\ Kazuki Fujimoto, Hirohito Kanamoto, Eiji Hanaoka, Kazuhisa Takahashi \\ Department of Orthopaedic Surgery, Graduate School of Medicine, Chiba University, Chiba, Japan
}

\begin{abstract}
Study Design: Retrospective case series.
Purpose: To examine the efficacy of TachoSil for vessel injury in 6 patients who underwent anterior lumbar fusion surgery (ALF). Overview of Literature: ALF for the lumbar spine has a high rate of success, although intraoperative concerns and iatrogenic complications are known, and injury of a major vessel is sometimes a complication. The efficacy of TachoSil, a fibrin-based hemostat, has been reported for several types of surgery; however, use of TachoSil for ALF surgery has not been described. Here, we report on the efficacy of TachoSil in 6 patients, who underwent ALF after vascular surgeons having difficulty in repairing vessels.

Methods: Two man and 4 women with average age of $50.8 \pm 10.9$ (mean \pm standard deviation) were diagnosed with a vertebral tumor (2 patients), L4 degenerative spondylolisthesis (2 patients), and L5 spondylolytic spondylolisthesis (2 patients) and underwent ALF. The blood vessels injured included the common iliac vein in 2 patients and a branch of a segmental artery from the aorta in 4 patients. We consulted a vascular surgeon to suture or repair the vessels during surgery, and although the vascular surgeon attempted to address the injuries, suturing or repair was not possible in these cases. For this reason, we used TachoSil to repair the injury in the vessels walls or to stop the bleeding.

Results: Time to pressure hemostasis using TachoSil was $34 \pm 12$ minutes, and total blood loss was 1,488 $\pm 1,711 \mathrm{~mL}$. Nevertheless, all vessel injuries were controlled by the use of TachoSil.

Conclusions: We recommend the use of TachoSil for vessel injuries that vascular surgeons cannot suture or repair during ALF surgery.
\end{abstract}

Keywords: TachoSil; A fibrin-based hemostat; Anterior lumbar fusion surgery; Blood

\section{Introduction}

Anterior lumbar fusion surgery (ALF) for the lumbar spine has a high rate of success, although intraoperative concerns and iatrogenic complications exist [1-3]. Injuries to major vessels or branches of segmental arteries from the aorta are sometimes a complication, and it may be dif- ficult to stop such bleeding. A wide range of local internal hemostatic agents has been developed to help achieve intracavity control of bleeding [4].

TachoSil (Nycomed, Linz, Austria) was developed from TachoComb (Nycomed) and TachoComb H (Nycomed) $[4,5]$. TachoComb contains equine collagen, bovine thrombin, bovine aprotinin, and human fibrinogen [5].

\footnotetext{
Received Jan 11, 2016; Revised Mar 25, 016; Accepted Apr 8, 2016

Corresponding author: Seiji Ohtori

Department of Orthopaedic Surgery, Graduate School of Medicine, Chiba University,

1-8-1 Inohana, Chuo-ku, Chiba 260-8670, Japan

Tel: +81-43-226-2117; Fax: +81-43-226-2116, E-mail: sohtori@faculty.chiba-u.jp
} 
TachoComb $\mathrm{H}$ is made human thrombin in place of bovine thrombin [5]. TachoSil, in turn, contains only human fibrinogen with equine collagen as a carrier [5], and it does not contain aprotinin or any other components of bovine origin [5].

Efficacy of TachoSil for several types of surgery has been reported; however, use of TachoSil for ALF surgery has not been described [6]. We report on the efficacy of TachoSil used in 6 patients who underwent ALF and the vascular surgeons were trying to repair difficult vessels.

\section{Materials and Methods}

We performed 496 surgeries on the lumbar spine from April of 2012 to December of 2015 in our medical center. ALF was performed in 184 patients. Among these, 12 patients had injury to blood vessels that included the common iliac vein or a segmental artery. Vascular injury of 6 of these 12 patients (50\%) could not be repaired by conventional techniques. Written informed consent was received from these 6 patients for their case reports to be published. Two man and 4 women underwent ALF as the conservative treatment was ineffective. The patients had an average age of $50.8 \pm 10.9$ years (mean \pm standard deviation $[\mathrm{SD}]$ ) and were diagnosed with a vertebral tumor (2 patients), L4 degenerative spondylolisthesis (2 patients), and L5 spondylolytic spondylolisthesis (2 patients) (Table 1). For L5 spondylolytic spondylolisthesis, open
ALF surgery using percutaneous pedicle screws (PPS) was conducted. For L4 degenerative spondylolisthesis, miniopen anterior retroperitoneal lumbar interbody fusion methods, such as oblique lumbar interbody fusion (OLIF) $[7,8]$ using PPS, were performed. For L2 and L3 vertebral tumor, L2 and L3 total en bloc spondylectomy was carried out, and for L3 vertebral tumor, open total vertebral resection with plate fixation was implemented.

The blood vessels injured included the common iliac vein in 2 patients and a branch of a segmental artery from the aorta in 4 patients. We consulted a vascular surgeon to suture or repair the vessels during surgery, and although the vascular surgeon attempted to suture or repair the injury, suturing or repair was not possible. For this reason, we used TachoSil to repair the injury to the wall of vessels or to stop the bleeding. There were no guidelines about the appropriate time to pressure hemostasis for spine surgery. Therefore, the time to pressure hemostasis using TachoSil was determined by each surgeon. We evaluated the time to pressure hemostasis using TachoSil, the total blood loss, and whether vessel injuries were controlled by using TachoSil. We confirmed hemostasis in the surgical field during surgery, and inserted a suction drain at the end of surgery. We also evaluated bleeding into the suction drain for 2 days after surgery. However, we did not evaluate hemostasis using additional radiological examinations after surgery.

Table 1. Patient background and total blood loss during surgery

\begin{tabular}{|c|c|c|c|c|c|c|}
\hline Diagnosis & Sex & $\begin{array}{l}\text { Age } \\
\text { (yr) }\end{array}$ & $\begin{array}{l}\text { Method of } \\
\text { surgery }\end{array}$ & $\begin{array}{l}\text { Total blood } \\
\text { loss (mL) }\end{array}$ & Injury site & $\begin{array}{c}\text { Time to pressure } \\
\text { hemostasis using } \\
\text { TachoSil (min) }\end{array}$ \\
\hline L3 vertebral tumor & Female & 39 & $\begin{array}{l}\text { Open spondylectomy } \\
\text { and plate fixation }\end{array}$ & 1,200 & $\begin{array}{l}\text { Branch of a segmental } \\
\text { artery from the aorta }\end{array}$ & 60 \\
\hline $\begin{array}{l}\text { L2, } 3 \text { vertebral } \\
\text { tumor }\end{array}$ & Male & 42 & $\begin{array}{l}\text { Total en bloc } \\
\text { spondylectomy }\end{array}$ & 750 & $\begin{array}{l}\text { Branch of a segmental } \\
\text { artery from the aorta }\end{array}$ & 10 \\
\hline $\begin{array}{l}\text { L4 degenerative } \\
\text { spondylolisthesis }\end{array}$ & Female & 68 & OLIF+PPS & 5,000 & $\begin{array}{l}\text { Branch of a segmental } \\
\text { artery from the aorta }\end{array}$ & 30 \\
\hline $\begin{array}{l}\text { L4 degenerative } \\
\text { spondylolisthesis }\end{array}$ & Female & 54 & OLIF+PPS & 850 & $\begin{array}{l}\text { Branch of a segmental } \\
\text { artery from the aorta }\end{array}$ & 30 \\
\hline $\begin{array}{l}\text { L5 spondylolytic } \\
\text { spondylolisthesis }\end{array}$ & Male & 60 & Open ALIF and PPS & 900 & Common iliac vein & 30 \\
\hline $\begin{array}{l}\text { L5 spondylolytic } \\
\text { spondylolisthesis }\end{array}$ & Female & 42 & Open ALIF and PPS & 230 & Common iliac vein & 45 \\
\hline Average & & $50.8 \pm 10.9$ & & $1,488 \pm 1,711$ & & $34 \pm 12$ \\
\hline
\end{tabular}

OLIF, oblique lumbar interbody fusion; PPS, percutaneous pedicle screws; ALIF, anterior lumbar interbody fusion. 


\section{Results}

Time to pressure hemostasis using TachoSil was from 10 to 60 minutes, with average time of $34 \pm 12$ minutes (mean \pm SD). Total blood loss was from 230 to $5,000 \mathrm{~mL}$, with average blood loss of $1,488 \pm 1,711$ (mean \pm SD) (Table 1). Fortunately, all vessel injuries were successfully controlled by using TachoSil. We did not observe bleeding after the surgery, did not find excess bleeding into the suction drain during the 2 days after surgery in 6 patients, and all patients recovered normally.

Fig. 1 shows the injury to the branch of the segmental artery from the aorta on the contralateral side after OLIF plus PPS surgery. We reoperated from the contralateral side, and were ultimately able stop the bleeding using TachoSil. Fig. 2 shows the injury to the branch of the segmental artery from the aorta during L2 and L3 total en bloc spondylectomy for the L2 and L3 vertebral tumor, and could stop the bleeding using TachoSil.

\section{Discussion}

In the current study, we present injury to the common iliac vein in 2 patients and a branch of a segmental artery from the aorta in 4 patients. All vessel injuries were controlled using TachoSil where a vascular surgeon could not suture or otherwise repair the vessel. From our experience, we recommend the use of TachoSil for such cases.

A systematic review and meta-analysis were used to assess the clinical and radiographic outcomes and complications of ALF versus TLIF. Fusion rates and clinical outcomes were comparable between ALF and TLIF. However, major blood vessel injury occurred significantly more frequently in the studied ALF cohort compared with the TLIF cohort (2.6\% vs. $0 \%$ ) [9]. This outcome was not surprising, given that the anterior technique involves exposure, manipulation, and mobilization of the iliac vessels, and increases the risk of bleeding via lacerations and thrombosis $[10,11]$. Vascular surgeons may help to
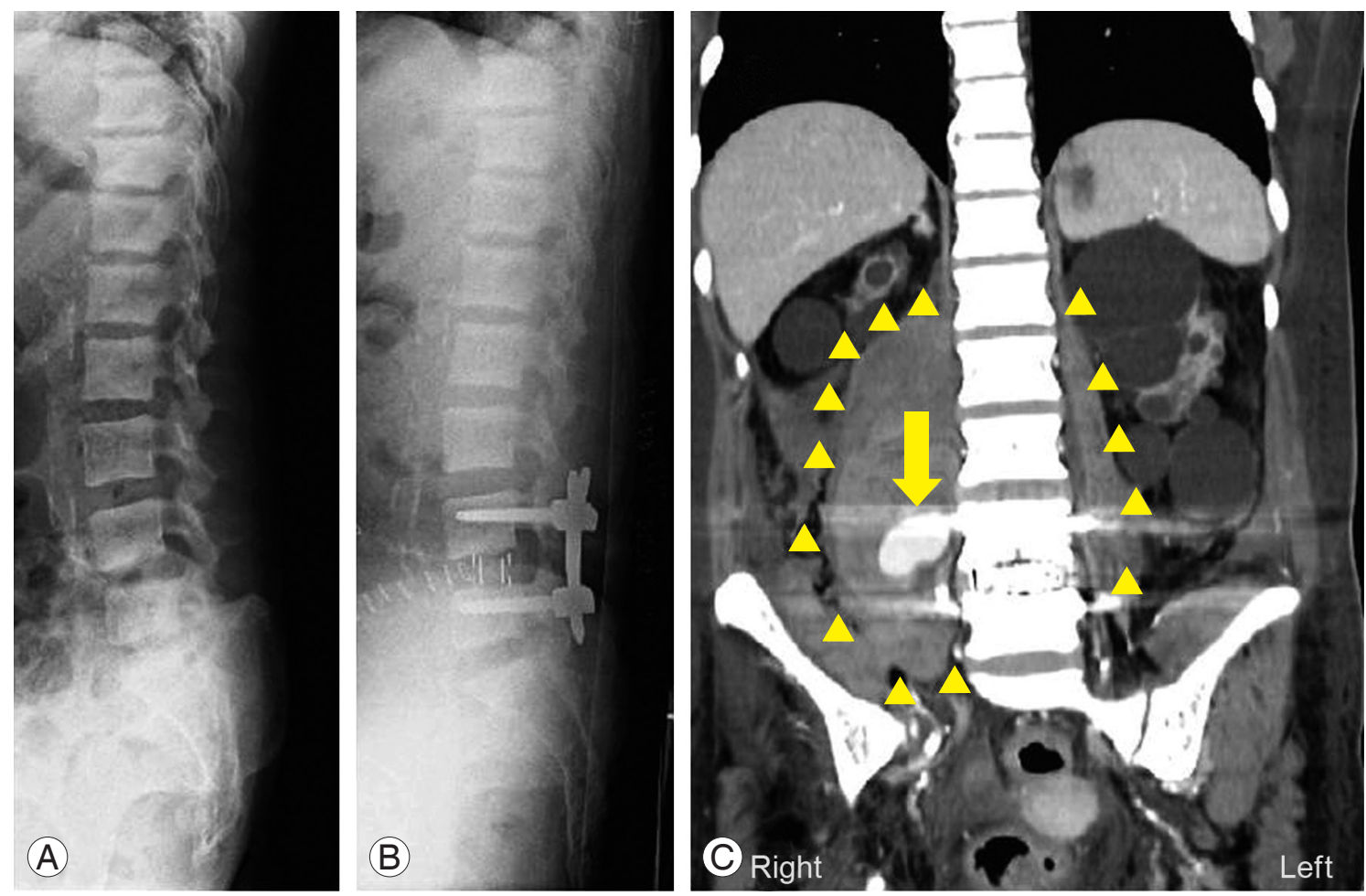

Fig. 1. Case presentation. A 68-year-old woman was diagnosed with $L 4$ degenerative spondylolisthesis. She underwent hemodialysis for 18 years. Profile X-ray imaging before surgery (A). Profile X-ray imaging after oblique lumbar interbody fusion before adding percutaneous pedicle screw surgery (B). The approach was from the left side. The patient showed acute anemia and motor weakness of her quadriceps after surgery, angiography showed pool of contrast medium (arrow) on the right, and swelling of the iliopsoas muscle (arrow heads). Branch of a segmental artery from the aorta was injured at $L 4$ vertebral level at contralateral side. We suspected that overcorrection tore off the vessel at the contralateral side (C). We reoperated on the patient, and used TachoSil for the vessel injury. 

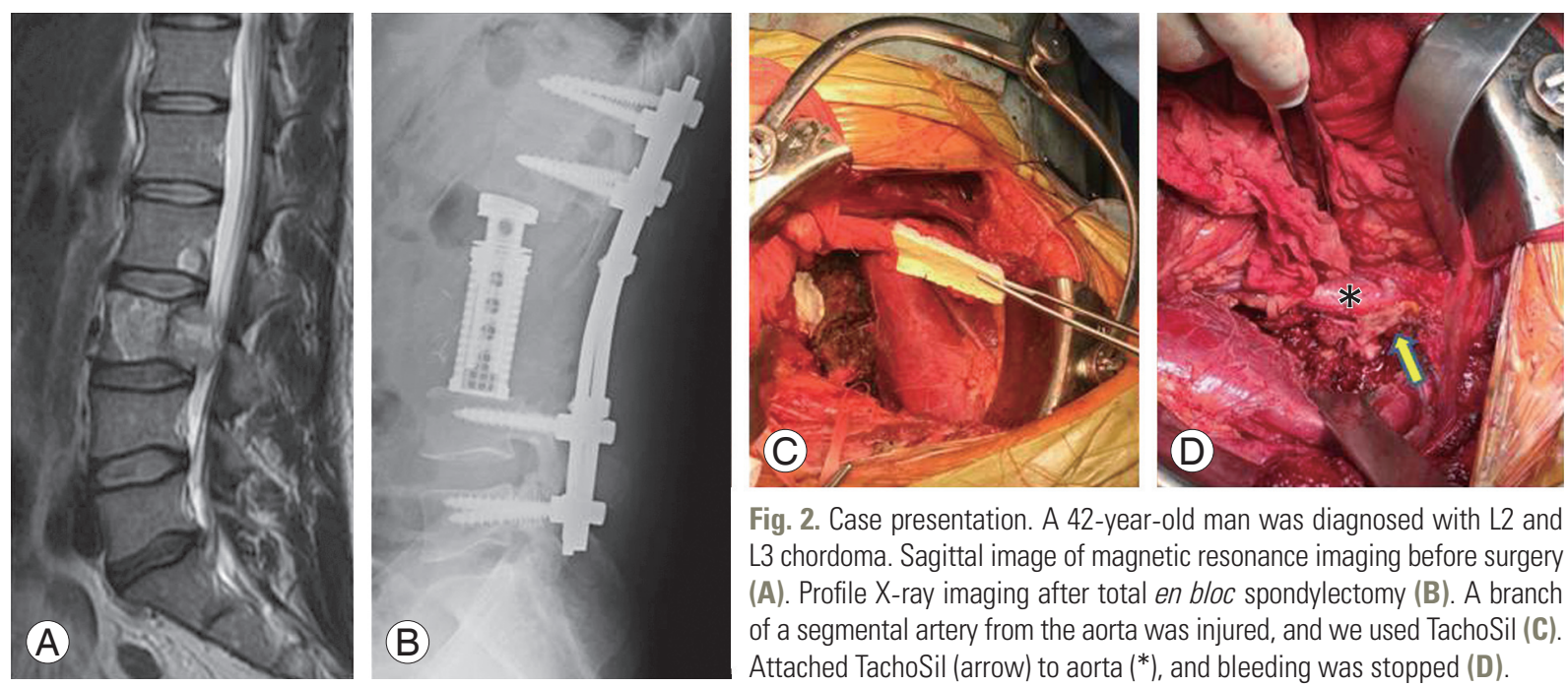

Fig. 2. Case presentation. A 42-year-old man was diagnosed with $\mathrm{L} 2$ and L3 chordoma. Sagittal image of magnetic resonance imaging before surgery (A). Profile X-ray imaging after total en bloc spondylectomy (B). A branch of a segmental artery from the aorta was injured, and we used TachoSil (C). Attached TachoSil (arrow) to aorta $\left({ }^{*}\right)$, and bleeding was stopped (D).

approach or repair the vessel injuries; however, suturing sometimes fails.

Until now hemostatics, used most commonly in spine surgery, have included oxidized, regenerated cellulose (Surgicel), gel sponges (Gelfoam, Spongostan), and collagen fleece (Avitene) [12]. Local hemostatics should be removed at the end of the surgery, but these materials are absorbable and so they are often left in place to avoid postoperative hematomas. Another hemostatic, bone wax which is a mixture of beeswax (70\%) and Vaseline (30\%) is not absorbable [12]. Recently, FloSeal (Baxter Healthcare, Deerfield, IL, USA) and Surgiflo (Ethicon, Somerville, NJ, USA), have been developed for bleeding control during spine surgery [13]. However, these agents are used for bleeding from the surgical field, and are not useful for vessel injuries during anterior lumbar spine surgery.

The efficacy and safety of TachoSil in patients with lung bleeding problems, liver resection, and splenectomy have been demonstrated, and there are also a few articles related to use of TachoSil in cardiovascular surgery [1416]. Excessive bleeding represents a significant risk for the patient in cardiovascular surgery. The safety and efficacy of TachoSil compared to conventional techniques to stop bleeding were examined [17]. In the study, 20 patients underwent TachoSil treatment and 22 patients received a conventional treatment using Surgicel (Ethicon, Johnson \& Johnson Medical, Neuchatel, Switzerland) [17]. In the TachoSil treated group, $75 \%$ of patients required transfusions versus $90 \%$ in the Surgicel treated group [17]. The investigators concluded that TachoSil might act as a superior alternative in various types of cardiac surgery to control bleeding and thereby reduce transfusion requirements [17]. To our knowledge, the efficacy of TachoSil for vessel injuries during anterior lumbar spine surgery has not been reported until now.

TachoSil is used for incidental durotomy, a common complication of lumbar spine surgery, with a prevalence ranging from less than $1 \%$ to $17 \%$, according to the series reviewed and the type of surgical procedure $[18,19]$. In March 2014, members of the Swiss, German, and Austrian neurosurgical and spine societies were asked to complete an online questionnaire regarding the management of incidental durotomy during and after lumbar spine surgery, and TachoSil/Spongostan with fibrin glue or a similar product $(79.4 \%)$ and single suture repair (69.7\%) were the treatments preferred by most surgeons [20]. In addition to repair incidental durotomy, we recommend the use of TachoSil for vessel injuries that vascular surgeons cannot suture or otherwise repair during ALF surgery.

\section{Conclusions}

We present the efficacy of TachoSil for injury to the common iliac vein in 2 patients and a branch of a segmental artery from the aorta in 4 patients. We recommend the use of TachoSil to treat difficult vessel injuries during anterior lumbar spine surgery.

\section{Conflict of Interest}

No potential conflict of interest relevant to this article was reported. 


\section{References}

1. Ishihara H, Osada R, Kanamori M, et al. Minimum 10-year follow-up study of anterior lumbar interbody fusion for isthmic spondylolisthesis. J Spinal Disord 2001;14:91-9.

2. Takahashi K, Kitahara H, Yamagata M, et al. Longterm results of anterior interbody fusion for treatment of degenerative spondylolisthesis. Spine (Phila Pa 1976) 1990;15:1211-5.

3. Tiusanen H, Seitsalo S, Osterman K, Soini J. Retrograde ejaculation after anterior interbody lumbar fusion. Eur Spine J 1995;4:339-42.

4. Dunn CJ, Goa KL. Fibrin sealant: a review of its use in surgery and endoscopy. Drugs 1999;58:863-86.

5. Toro A, Mannino M, Reale G, Di Carlo I. TachoSil use in abdominal surgery: a review. J Blood Med 2011; 2:31-6.

6. Rickenbacher A, Breitenstein S, Lesurtel M, Frilling A. Efficacy of TachoSil a fibrin-based haemostat in different fields of surgery: a systematic review. Expert Opin Biol Ther 2009;9:897-907.

7. Ohtori S, Mannoji C, Orita S, et al. Mini-open anterior retroperitoneal lumbar interbody fusion: oblique lateral interbody fusion for degenerated lumbar spinal kyphoscoliosis. Asian Spine J 2015;9:565-72.

8. Ohtori S, Orita S, Yamauchi K, et al. Mini-open anterior retroperitoneal lumbar interbody fusion: oblique lateral interbody fusion for lumbar spinal degeneration disease. Yonsei Med J 2015;56:1051-9.

9. Phan K, Thayaparan GK, Mobbs RJ. Anterior lumbar interbody fusion versus transforaminal lumbar interbody fusion: systematic review and meta-analysis. $\mathrm{Br}$ J Neurosurg 2015;29:705-11.

10. Chiriano J, Abou-Zamzam AM Jr, Urayeneza O, Zhang WW, Cheng W. The role of the vascular surgeon in anterior retroperitoneal spine exposure: preservation of open surgical training. J Vasc Surg 2009;50:148-51.

11. Brau SA, Delamarter RB, Schiffman ML, Williams LA, Watkins RG. Vascular injury during anterior lumbar surgery. Spine J 2004;4:409-12.
12. Schonauer C, Tessitore E, Barbagallo G, Albanese V, Moraci A. The use of local agents: bone wax, gelatin, collagen, oxidized cellulose. Eur Spine J 2004;13 Suppl 1:S89-96.

13. Landi A, Gregori F, Marotta N, Delfini R. Efficacy, security, and manageability of gelified hemostatic matrix in bleeding control during thoracic and lumbar spine surgery: FloSeal versus Surgiflo. J Neurol Surg A Cent Eur Neurosurg 2016;77:139-43.

14. Maisano F, Kjaergard HK, Bauernschmitt R, et al. TachoSil surgical patch versus conventional haemostatic fleece material for control of bleeding in cardiovascular surgery: a randomized controlled trial. Eur J Cardiothorac Surg 2009;36:708-14.

15. Rena O, Papalia E, Mineo TC, et al. Air-leak management after upper lobectomy in patients with fused fissure and chronic obstructive pulmonary disease: a pilot trial comparing sealant and standard treatment. Interact Cardiovasc Thorac Surg 2009;9:973-7.

16. Tagliabue F, D’Angelo C, Zuccon W, Giorgetta C, Gambarini F, Bonandrini L. Use of Tachosil in splenectomy in patients with clotting and blood composition disorders. Minerva Chir 2007;62:73-8.

17. Alizadeh Ghavidel A, Mirmesdagh Y, Samiei N, Gholampour Dehaki M. Haemostatic role of tachosil surgical patch in cardiac surgery. J Cardiovasc Thorac Res 2014;6:91-5.

18. Deyo RA, Cherkin DC, Loeser JD, Bigos SJ, Ciol MA. Morbidity and mortality in association with operations on the lumbar spine: the influence of age, diagnosis, and procedure. J Bone Joint Surg Am 1992;74: 536-43.

19. Kalevski SK, Peev NA, Haritonov DG. Incidental Dural Tears in lumbar decompressive surgery: incidence, causes, treatment, results. Asian J Neurosurg 2010;5:54-9.

20. Gautschi OP, Stienen MN, Smoll NR, Corniola MV, Tessitore E, Schaller K. Incidental durotomy in lumbar spine surgery: a three-nation survey to evaluate its management. Acta Neurochir (Wien) 2014;156:1813-20. 